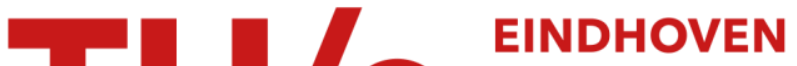 UNIVERSITY OF TECHNOLOGY
}

\section{Temperature- and density-dependent channel potentials in high-mobility organic field-effect transistors}

\section{Citation for published version (APA):}

Kemerink, M., Hallam, T., Lee, M. J., Zhao, N., Caironi, M., \& Sirringhaus, H. (2009). Temperature- and density-dependent channel potentials in high-mobility organic field-effect transistors. Physical Review B, (11), 115325-1/5. [115325]. https://doi.org/10.1103/PhysRevB.80.115325

DOI:

10.1103/PhysRevB.80.115325

Document status and date:

Published: 01/01/2009

\section{Document Version:}

Publisher's PDF, also known as Version of Record (includes final page, issue and volume numbers)

\section{Please check the document version of this publication:}

- A submitted manuscript is the version of the article upon submission and before peer-review. There can be important differences between the submitted version and the official published version of record. People interested in the research are advised to contact the author for the final version of the publication, or visit the $\mathrm{DOI}$ to the publisher's website.

- The final author version and the galley proof are versions of the publication after peer review.

- The final published version features the final layout of the paper including the volume, issue and page numbers.

Link to publication

\section{General rights}

Copyright and moral rights for the publications made accessible in the public portal are retained by the authors and/or other copyright owners and it is a condition of accessing publications that users recognise and abide by the legal requirements associated with these rights.

- Users may download and print one copy of any publication from the public portal for the purpose of private study or research.

- You may not further distribute the material or use it for any profit-making activity or commercial gain

- You may freely distribute the URL identifying the publication in the public portal.

If the publication is distributed under the terms of Article 25fa of the Dutch Copyright Act, indicated by the "Taverne" license above, please follow below link for the End User Agreement:

www.tue.nl/taverne

Take down policy

If you believe that this document breaches copyright please contact us at:

openaccess@tue.nl

providing details and we will investigate your claim. 


\title{
Temperature- and density-dependent channel potentials in high-mobility organic field-effect transistors
}

\author{
M. Kemerink, * T. Hallam, M. J. Lee, N. Zhao, M. Caironi, and H. Sirringhaus \\ Cavendish Laboratory, J. J. Thomson Avenue, Cambridge CB3 OHE, United Kingdom
}

(Received 20 April 2009; revised manuscript received 4 August 2009; published 25 September 2009)

\begin{abstract}
The density-dependent charge-carrier mobility in high-mobility organic field-effect transistors is investigated by simultaneous measurements of the channel potential and the transfer characteristics. By working under ultrahigh-vacuum conditions extrinsic effects due to $\mathrm{H}_{2} \mathrm{O}$ traces could be eliminated. The shape of the channel potential is inconsistent with a density-independent mobility. We find that the variable range hopping model as derived by Vissenberg and Matters for an exponential density of states [Phys. Rev. B 57, 12964 (1998)] consistently describes the data.
\end{abstract}

DOI: $10.1103 /$ PhysRevB.80.115325

PACS number(s): $85.30 . T v$, 72.80.Le, 68.37.Ps

\section{INTRODUCTION}

Over the years, a large number of fundamentally different models has been proposed to describe the charge transport in organic semiconducting materials. For amorphous, lowmobility materials such as poly(phenylene-vinylene)s (Ref. 1) there seems to be consensus on a description in terms of variable range hopping (VRH) of polaronic charges between localized states in a density of states (DOS) that is broadened by energetic disorder. ${ }^{2}$ The shape of the dominant lowenergy tail of the DOS is usually assumed to be either exponential or Gaussian. For (poly)crystalline high-mobility materials such as poly(thiophene)s, oligo(acene)s, and oligo(thiophene)s, the situation is less clear and consensus on the preferred model(s) has not been reached. ${ }^{3}$ For such materials, the various models can roughly be separated in three "classes," each based on different underlying assumptions. For example, the VRH model by Vissenberg and Matters (VM), which is based on the same assumptions of localized state hopping as commonly used for low-mobility materials, has successfully been applied to describe the densitydependent carrier mobility in amorphous poly(3hexylthiophene) by Tanase et al..$^{4,5}$ On the other hand, it has been argued that for sufficiently low disorder, i.e., for sufficiently narrow DOS, states above (or below, for holes) a certain threshold energy become delocalized, giving rise to a situation where charges in low-energy states are effectively trapped and conductivity is dominated by charges in the delocalized states. This "mobility edge" (ME) or "multiple trapping and release" model was originally proposed for organic materials by Horowitz and Delannoy ${ }^{6}$ and later advocated by Salleo et al. ${ }^{7}$ on basis of measurements on a regioregular poly(thiophene). Finally, depending on the degree of (poly)crystallinity and the coupling between neighboring crystallites, the long-range conductivity may actually be dominated by intergrain transport, rather than by intragrain transport. ${ }^{8,9}$ This seems, e.g., to be the case for evaporated small molecule films. ${ }^{10,11}$

Simultaneously, we note that so far virtually all experimental evidence for the above mobility models has been obtained from the electrical characteristics of organic fieldeffect transistors (OFETs) alone. However, scanning Kelvin probe microscopy (SKPM) offers the unique possibility to look inside the operational device by measuring the electrostatic surface potential on top of the active layer. For thin $(<100 \mathrm{~nm})$ layers, and depending on the spatial and energetic resolutions of the measurement scheme employed, this can be an excellent measure of the actual channel potential.

Smits et al. ${ }^{12}$ performed a room-temperature SKPM study to channel potentials in OFETs and reported good agreement between measured potentials and a theoretical model based on variable range hopping. However, the experimental resolution and the associated rescaling procedure prevented a detailed comparison between various models. Bürgi et al. ${ }^{13} \mathrm{em}$ ployed a high-resolution SKPM scheme to similar devices but interpreted the results in a model that takes the chargedensity dependence of the mobility into account in a phenomenological manner. ${ }^{14}$

In this work, we use high resolution, variable-temperature SKPM to measure the electrostatic potential distribution $V(x)$ across the OFET channel while simultaneously measuring the electrical device characteristics. We show that the density dependence of the mobility gives rise to a $V(x)$ that fundamentally differs from that of a constant mobility. Moreover, the temperature dependence of $V(x)$ can be used to discriminate between various mobility models.

\section{EXPERIMENT}

The OFET devices used in this work were fabricated in dry nitrogen by spincoating a $5 \mathrm{mg} / \mathrm{ml}$ solution of poly(2,5-bis(3-alkylthiophen-2-yl)thieno[3,2-b]thiophene) (pBTTT) in 1,2-dichlorobenzene on precleaned $\mathrm{Si} / \mathrm{SiO}_{2}(300$ $\mathrm{nm}$ oxide) substrates. ${ }^{15}$ Before spin coating, the substrates were exposed to octyltrichlorosilane (OTS) vapor for $3 \mathrm{~h}$. The used parameters $(2000 \mathrm{rpm})$ led to a film thickness of roughly $30 \mathrm{~nm}$. To improve crystallinity, films where annealed at $170{ }^{\circ} \mathrm{C}$ for $10 \mathrm{~min}$ under nitrogen atmosphere. Afterward, $\mathrm{Au}$ top contacts where thermally evaporated at $0.5 \AA / \mathrm{s}$ through a shadow mask to yield devices with channel length $L=20 \mu \mathrm{m}$ and width $W=1 \mathrm{~mm}$. The UHV SKPM apparatus was an Omicron variable temperature atomic force microscope (VT-AFM) used in noncontact mode at pressures below $10^{-8}$ mbar. The samples were cooled in UHV via a thermal link to the internal He flow cryostat, giving an overall thermal stability at the sample 
stage of about $1 \mathrm{~K}$. Tip oscillation was controlled via a Nanonis OC-4 phase-locked loop. The built-in Kelvin controller of the OC- 4 was used to measure surface potentials using the frequency modulation technique. ${ }^{13,16}$ For thin, intrinsic semiconductors, the surface potential closely follows the potential in the channel. ${ }^{13}$ In order to allow the measurement of potentials in excess of $\pm 10 \mathrm{~V}$, the Kelvin controller was connected to the tip via a $20 \times$ voltage amplifier. The used tips were PtIr coated $\mathrm{Si}$, with a cantilever spring constant of $k \approx 2 \mathrm{~N} / \mathrm{m}$ and a resonance frequency $f_{\text {res }} \approx 70 \mathrm{kHz}$. In the measurements shown below, the overall spatial and potential resolutions were better than $100 \mathrm{~nm}$ and $10 \mathrm{mV}$, respectively.

\section{MODELS}

Below, electrostatic potentials and linear mobilities will be compared to the predictions of various mobility models that have been described in detail in the literature. For completeness, a concise summary of the main formulas is provided in this section.

Assuming variable range hopping in an exponential density of states with characteristic width $T_{0}$, VM derived the following expression for the linear mobility as a function of gate voltage, ${ }^{4}$

$$
\begin{aligned}
\mu= & \frac{\sigma_{0}}{q}\left[\frac{\pi\left(T_{0} / T\right)^{3}}{(2 \alpha)^{3} B_{c} \Gamma\left(1-T_{0} / T\right) \Gamma\left(1+T_{0} / T\right)}\right]^{T / T_{0}} \\
& \times\left[\frac{\left(C V_{G}^{\prime}\right)^{2}}{2 k_{B} T_{0} \varepsilon_{S}}\right]^{T_{0} / T-1} .
\end{aligned}
$$

In Eq. (1), $\sigma_{0}$ is a conductivity prefactor and $B_{c} \approx 2.8$ the number of bonds per site in the percolating cluster and $\alpha^{-1}$ the decay length of the localized wave function. Further, $q$ is the elementary charge, $k_{B}$ the Boltzmann constant, $T$ the absolute temperature, $\varepsilon_{S}$ the dielectric constant of the semiconductor, and $C$ the areal capacitance of the dielectric layer. Finally, $V_{G}^{\prime}$ is the effective local gate bias, i.e., $V_{G}^{\prime}=V_{G}-V_{t h}-V(x)$ with $V_{G}$ the applied gate voltage, $V_{t h}$ the threshold voltage, and $V(x)$ the local electrostatic potential in the channel. The gamma functions in Eq. (1) can be approximated to yield ${ }^{5}$

$$
\mu=\frac{\sigma_{0}}{q}\left[\frac{\pi\left(T_{0} / T\right)^{4} \sin \left(\pi T / T_{0}\right)}{(2 \alpha)^{3} B_{c}}\right]^{T / T_{0}}\left[\frac{\left(C V_{G}^{\prime}\right)^{2}}{2 k_{B} T_{0} \varepsilon_{S}}\right]^{T_{0} / T-1} .
$$

In the ME model an exponential distribution of traps, with characteristic temperature $T_{0}$ and total density $g_{0}$, is assumed to exist below a band in which carriers are mobile with mobility $\mu_{0}$. The effective mobility in the channel then becomes $^{7,17}$

$$
\mu=\mu_{0} \frac{N_{m o b}}{N_{\text {tot }}}
$$

with $N_{m o b}$ the number of mobile carriers, which is related to the Fermi level $E_{F}$ via
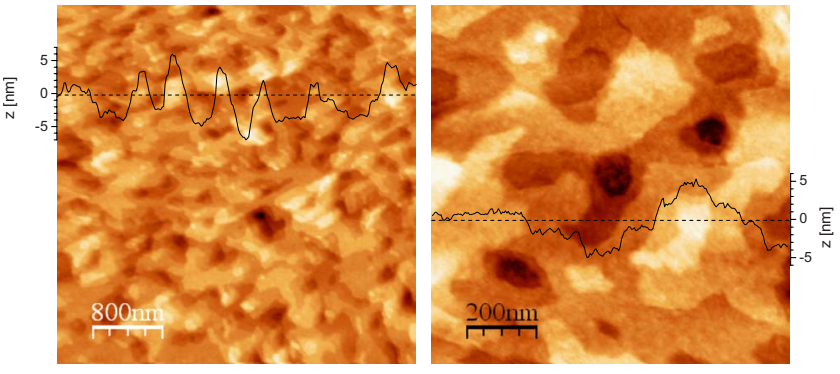

FIG. 1. (Color online) Topographical AFM images of a pBTTT film in the middle of the transistor channel. Scan sizes are $4 \times 4$ and $1 \times 1 \mu \mathrm{m}$ in panels (a) and (b); the total vertical scales are 21 and $14 \mathrm{~nm}$, respectively. The solid lines are height profiles along the sections indicated by the dashed lines. $T=250 \mathrm{~K}$.

$$
N_{m o b}=\int_{-\infty}^{0} D(E) f\left(E_{F}, E\right) d E
$$

with $D$ the total DOS and $f$ the Fermi-Dirac distribution function. The Fermi energy is related to the total number of carriers in the channel $N_{t o t}$, via the relation

$$
N_{\text {tot }}=\frac{C\left|V_{G}^{\prime}\right|}{h}=\int_{-\infty}^{\infty} D(E) f\left(E_{F}, E\right) d E
$$

with $h \approx 1 \mathrm{~nm}$ the thickness of the accumulation layer. ${ }^{7}$ In Eqs. (4) and (5) states below $E=0$ are mobile whereas states above $E=0$ are assumed to have a negligible mobility.

Equations (2)-(5) give rise to a mobility that depends on the charge density and hence on the local effective gate bias. In case of a non-negligible source-drain bias, this effective gate bias is not constant throughout the channel and the mobility becomes position dependent and needs to be determined self-consistently together with the channel potential $V(x)$. In this work we numerically obtained $V(x)$ using an iterative procedure, regarding the channel as a series network of resistive elements. For the VM model this is equivalent to the analytical expressions derived in Ref. 12. For a constant mobility, these expressions reduce to

$$
V(x)=V_{G}+\sqrt{\frac{x}{L}\left[\left(V_{D}-V_{G}\right)^{2}-V_{G}^{2}\right]+V_{G}^{2}} .
$$

Here, hole transport is assumed, i.e., $V_{G}<0$. Gate $\left(V_{G}\right)$ and drain $\left(V_{D}\right)$ biases are respective to the source bias $\left(V_{S}=0\right)$. In case of a nonzero threshold voltage, $V_{G}$ in Eq. (6) has to be replaced by $V_{G}-V_{t h}$.

It should be noted that Eq. (6) does not depend on the absolute value of the mobility. This statement also holds for a density-dependent mobility in which case only the relative change in the mobility with density matters for the shape of $V(x)$. For the VM model, this dependency is characterized by the parameter $\beta=2 T_{0} / T^{12}$

\section{RESULTS AND DISCUSSION}

In Fig. 1(a) a typical large area topography of the pBTTT layer in the middle of the transistor channel is shown. The 


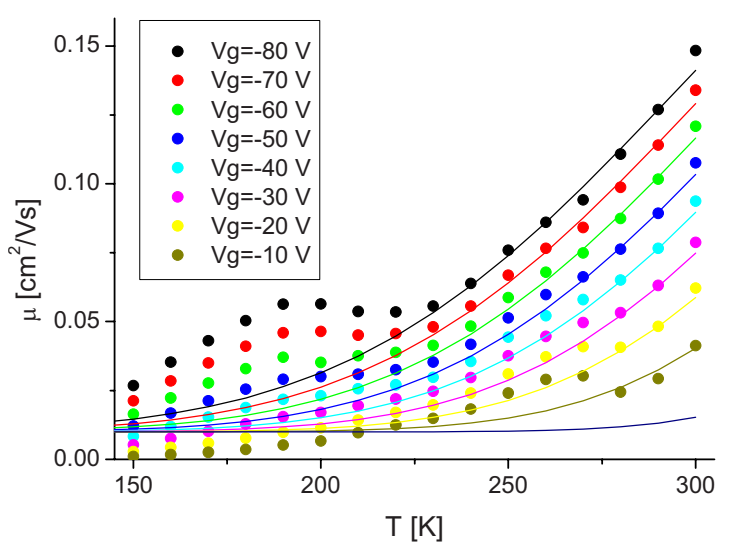

FIG. 2. (Color online) Linear mobility vs temperature for different gate biases, measured ex situ. Symbols denote experimental data; solid lines are fits to the VM model. Parameters used are $T_{0}=410 \mathrm{~K}, \alpha^{-1}=1.9 \AA$, and $\sigma_{0}=5.7 \times 10^{7} \mathrm{~S} / \mathrm{m}$.

film surface consists of monolayer terraces of densely packed pBTTT molecules, ${ }^{18}$ with a typical lateral size of a few hundred nm. Panel (b) shows a small area scan, revealing clear monolayer steps, approximately $2-2.5 \mathrm{~nm}$ in height, between for the rest smooth and featureless terraces. The step height slightly above $2 \mathrm{~nm}$ is consistent with a lamellar stacking due to the alkyl (C14) side chains along the $a$ axis. ${ }^{15,18}$ The most important feature of Fig. 1 is the total absence of topographical defects and the long-range smoothness. On average, we found that only a very minor fraction of large area scans such as Fig. 1(a) showed signs of topographical defects, originating from extrinsic sources during fabrication. This illustrates the high quality of the films. It is important to stress, however, that topographical smoothness does not automatically imply good electrical connectivity between the constituent crystalline domains. In the discussion of the measured electrostatic potentials we will return to this subject.

The temperature-dependent linear mobility of staggered (top contact, bottom gate) pBTTT devices measured ex situ in a vacuum chamber (chamber pressure $<1 \times 10^{-5}$ torr) is plotted in Fig. 2 for different gate biases. As anticipated the mobility drops with decreasing temperature and decreasing absolute gate bias, i.e., decreasing carrier concentration. However, between 225 and $150 \mathrm{~K}$ anomalous behavior is observed. It prevents an adequate fit to any of the mobility models discussed above as these predict monotonous behavior. This is illustrated by the solid lines which are a best fit to the VM model in the range 300-225 K. We note that similar behavior has been observed in thiophene derivatives before $^{7,8,19}$ and has been attributed to either structural relaxation in the polymer, ${ }^{7}$ multiple transport regimes. ${ }^{8}$ or the presence of trace (supercooled) $\mathrm{H}_{2} \mathrm{O} \cdot{ }^{19,20}$ Since the feature is absent in the data taken under UHV conditions, see Fig. 3, we support the latter interpretation. We note that this feature is also absent in bottom contact, top gate devices, where an organic dielectric is used instead of OTS-treated $\mathrm{SiO}_{2}{ }^{21} \mathrm{As}$ the accumulation layer in top gate devices is not accessible by SKPM we will not discuss these devices further. In either case, the results in Fig. 2 indicate that meaningful mobilities of poly(thiophene)s on $\mathrm{SiO}_{2}$ gate dielectrics can experimentally only be obtained when care is taken to remove all traces of $\mathrm{H}_{2} \mathrm{O}$.

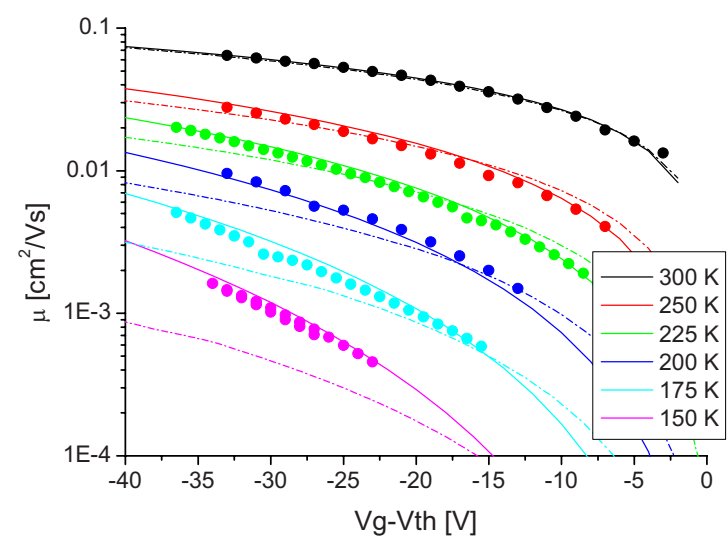

FIG. 3. (Color online) Linear mobility vs effective gate bias for different temperatures, measured in situ under UHV conditions. Symbols denote experimental data; solid and dashed lines are fits to the VM and ME models, respectively. Parameters used are $T_{0}=410 \mathrm{~K}, \alpha^{-1}=3.2 \AA$, and $\sigma_{0}=6.4 \times 10^{6} \mathrm{~S} / \mathrm{m}$ for the $\mathrm{VM}$ model and $T_{0}=510 \mathrm{~K}, g_{0}=3.5 \times 10^{26} \mathrm{~m}^{-3}$, and $\mu_{0}=2 \mathrm{~cm}^{2} / \mathrm{Vs}$ for the ME model.

The $\mathrm{H}_{2} \mathrm{O}$-related anomaly in the channel mobility is absent in the in situ measured data plotted in Fig. 3. Unlike for the ex situ data, it is in this case possible to make a consistent fit to the VM model in the full temperature range 150-300 K. Note that the extracted parameters are similar but not identical to those extracted from the ex situ measurements. This suggests that the presence of $\mathrm{H}_{2} \mathrm{O}$, or $\mathrm{H}_{2} \mathrm{O}$-related ionic species in or on the $\mathrm{SiO}_{2},{ }^{22}$ adversely affects the charge transport at all temperatures and not just in the temperature window in which the anomaly is observed. However, in both cases, the obtained parameters are reasonably close to previous findings. 5,7

At a single, constant temperature, the VM and ME models cannot be distinguished, as illustrated by the black solid and dashed-dotted lines in Fig. 3 and both models match the data well. However, it turns out to be impossible to obtain a good fit of the magnitude of the mobility over the full temperature and gate bias range with the ME model using a single set of parameters. The parameters used in Fig. 3 are optimized to yield a good fit at $300 \mathrm{~K}$; an equally good fit can be obtained at any other temperature but at the cost of a lesser fit at other temperatures. The increased gate voltage dependence at lower temperatures in the VM model reflects the downward shift of the transport level with decreasing temperature that is typical for VRH models. Consequently, a change in Fermi energy, due to a change in $V_{g}$, has a larger effect on $\mu$ at lower temperatures. In contrast, in the ME model the transport level is fixed by definition. This result strongly suggests that the concept of a fixed, temperature and densityindependent transport level or mobility edge, in combination with an exponential density of states, is inapplicable to the present devices. This notion is confirmed by the SKPM measurements of the channel potential that are discussed below.

Figure 4 shows the surface potential of the channel region of the pBTTT transistors for two different $V_{G}$ and $V_{D}$ configurations. The top contacts are sitting at $x<0$ and $x>19 \mu \mathrm{m}$. The minor difference between applied and measured voltages on the contacts is due to the work function 

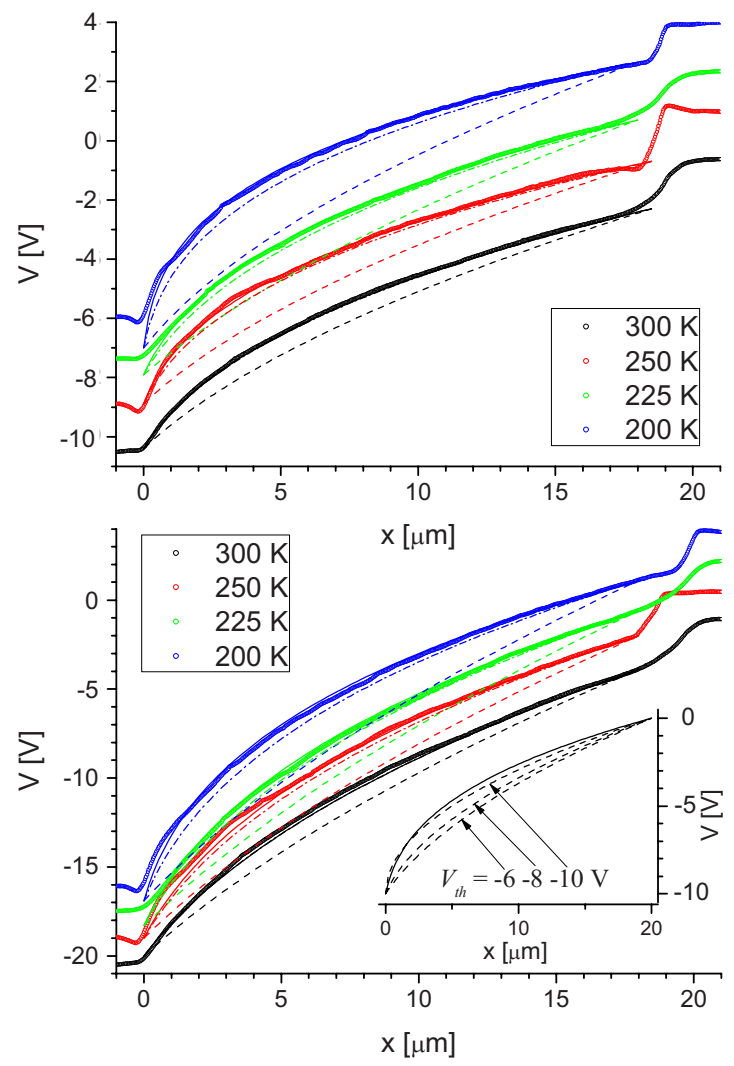

FIG. 4. (Color online) Electrostatic potentials vs position in the pBTTT OFET channel for different temperatures. Symbols are experimental data; solid, dashed-dotted, and dashed lines are calculated from the VM, ME, and constant mobility models, respectively. Parameters are given in the caption to Fig. 3. Top panel: $V_{G}=-20 \mathrm{~V}, V_{D}=-10$, and $V_{S}=0 \mathrm{~V}$; bottom panel: $V_{G}=-40 \mathrm{~V}$, $V_{D}=-20$, and $V_{S}=0 \mathrm{~V}$. Curves are offset for clarity. Inset: Calculated $V(x)$ from VM model $\left(V_{t h}=-6 \mathrm{~V}\right.$, solid line) and constant mobility ( $V_{t h}=-6,-8$, and $-10 \mathrm{~V}$ as indicated, dashed lines). $T=250 \mathrm{~K}$, other parameters as in main panel.

difference between the PtIr tip and the Au contacts. The voltage drop of about $10 \%$ of the applied source-drain bias in the vicinity of the injecting contact $(x \approx 19 \mu \mathrm{m})$ indicates a minor contact resistance. ${ }^{23}$ The overall smoothness of the curves and the absence of major, localized voltage drops indicate a good electrical contact between crystalline domains in the film. If the latter were dominant, a stepwise dropping potential would result. ${ }^{10,11}$ In other words, the device current is limited by bulk properties of the organic material (at the dielectric interface) rather than by intergranular contact effects. This is an explicit justification for the use of the VM and ME models, and the reason for not fitting our data to models that describe device performance in terms of grainboundary effects. In previous SKPM experiments, on other materials, ${ }^{10,11}$ such effects often dominated the response, prohibiting an evaluation of the shape of $V(x)$ in terms of bulk properties.

Using the parameters that were obtained in the fitting of the data in Fig. 3, the lines in Figs. 4(a) and 4(b) were calculated. It is important to stress that these calculations do not contain any additional free parameters, other than the values of $V(x)$ at the contacts. Clearly, the VM model (solid lines) gives an excellent description of the experiments at all temperatures. At $300 \mathrm{~K}$ the ME model (dashed-dotted lines) is virtually indistinguishable from the VM model but at reduced temperatures a small but systematic deviation occurs. Intuitively, it may seem surprising that the ME model still gives an acceptable description of $V(x)$ at low $T$, despite its poor predictions for $\mu$ at lower temperatures (Fig. 3). This is a consequence of the fact that the shape of $V(x)$ only depends on the relative density dependence of the carrier mobility and not on its magnitude, as discussed above. The underestimation of the curvature in $V(x)$ at low $T$ by the ME model is consistent with the deviations for this model in Fig. 3, which can be understood as follows. First, the effective gate bias, $V_{G}-V_{t h}-V(x)$, decreases toward the end of the channel, i.e., toward small $x$ in Fig. 4. Thus, the hole density and concomitantly the local mobility decreases upon approaching the drain. Second, current conservation requires the product of mobility, density, and electric field to be constant throughout the channel. A locally lower mobility therefore needs to be compensated by a locally larger field, i.e., a larger $\partial V / \partial x$. Consequently, the stronger the density dependence in the mobility, the larger the fraction of the total applied bias that drops in the region of lowest density, and the larger the curvature $\partial V^{2} / \partial x^{2}$ of $V(x)$ must be. From Fig. 3 it follows that the ME model underestimates the density dependence of the mobility at reduced $T$. So, according to the preceding argument, the ME model should result in an underestimation of $\partial V^{2} / \partial x^{2}$ at these temperatures. Indeed, this deviation is observed in Fig. 4.

A second important conclusion that can be drawn from Fig. 4 is that the measured $V(x)$ has a fundamentally different shape than calculated from Eq. (6) for a constant mobility, as shown by the dashed lines. This is further illustrated in the inset of Fig. 4(b) where a typical potential curve calculated from the VM model (solid line) is compared to Eq. (6) (dashed lines). Even when the threshold voltage $V_{t h}$ is freely varied in the latter model, the correct shape cannot be reproduced. Also the measured temperature dependence in the shape of $V(x)$ is fundamentally incompatible with a constant mobility model. Therefore, these measurements form an independent and direct confirmation of the density dependence of the mobility.

Finally, we should return to the subject of distinguishing transport models on basis of measurements as presented in this paper. Although we have shown that under the assumption of an exponential DOS the VM model gives a more consistent description of the experiments than the ME model, this conclusion does depend on the exact shape of the DOS. E.g., when a stretched exponential DOS $\left\{\sim \exp \left[-\left(E / k_{B} T_{0}\right)^{\beta}\right]\right\}$ is used in the ME model, the quality of the fitting can be drastically improved for $\beta \approx 0.7$, although the description does not become as accurate as that of the ME model. For other, more complex shapes of the DOS, the models are likely to be indistinguishable.

\section{SUMMARY}

We have performed simultaneous measurements of the electrostatic channel potential and the charge-carrier mobility 
as a function of temperature and gate bias in a high-mobility organic field-effect transistor. By working in UHV spurious effects of absorbed $\mathrm{H}_{2} \mathrm{O}$ could be avoided. The results allow us to evaluate different transport models. We find that the data can consistently be described by a model based on variable range hopping in an exponential density of states, as proposed by Vissenberg and Matters. ${ }^{4}$ Description of the data in terms of a mobility edge model requires the use of a more complicated density of states. In either case, the shape of the channel potential and its dependence on temperature form an independent and direct proof for the density dependence of the mobility.

\section{ACKNOWLEDGMENT}

We gratefully acknowledge M. Heeney and I. McCulloch for providing the polymer material and R. Coehoorn for stimulating discussions.
*Permanent address: Dept. of Applied Physics, Eindhoven University of Technology, 5600 MB Eindhoven, The Netherlands; m.kemerink@tue.nl

${ }^{1}$ P. W. M. Blom, C. Tanase, D. M. de Leeuw, and R. Coehoorn, Appl. Phys. Lett. 86, 092105 (2005).

${ }^{2}$ For a review, see, e.g., R. Coehoorn, W. F. Pasveer, P. A. Bobbert, and M. A. J. Michels, Phys. Rev. B 72, 155206 (2005).

${ }^{3}$ H. Sirringhaus, Adv. Mater. 17, 2411 (2005).

${ }^{4}$ M. C. J. M. Vissenberg and M. Matters, Phys. Rev. B 57, 12964 (1998).

${ }^{5}$ C. Tanase, E. J. Meijer, P. W. M. Blom, and D. M. de Leeuw, Phys. Rev. Lett. 91, 216601 (2003).

${ }^{6}$ G. Horowitz and P. Delannoy, J. Appl. Phys. 70, 469 (1991).

${ }^{7}$ A. Salleo, T. W. Chen, A. R. Völkel, Y. Wu, P. Liu, B. S. Ong, and R. A. Street, Phys. Rev. B 70, 115311 (2004).

${ }^{8}$ G. Horowitz, M. E. Hajlaoui, and R. Hajlaoui, J. Appl. Phys. 87, 4456 (2000).

${ }^{9}$ R. A. Street, J. E. Northrup, and A. Salleo, Phys. Rev. B 71, 165202 (2005).

${ }^{10}$ K. P. Puntambekar, P. V. Pesavento, and C. D. Frisbie, Appl. Phys. Lett. 83, 5539 (2003).

${ }^{11}$ P. Annibale, C. Albonetti, P. Stoliar, and F. Biscarini, J. Phys. Chem. A 111, 12854 (2007).

${ }^{12}$ E. C. P. Smits, S. G. J. Mathijssen, M. Cölle, A. J. G. Mank, P. A. Bobbert, P. W. M. Blom, B. de Boer, and D. M. de Leeuw, Phys. Rev. B 76, 125202 (2007).
${ }^{13}$ L. Bürgi, H. Sirringhaus, and R. H. Friend, Appl. Phys. Lett. 80, 2913 (2002).

${ }^{14}$ L. Bürgi, T. J. Richards, M. Chiesa, R. H. Friend, and H. Sirringhaus, Synth. Met. 146, 297 (2004).

${ }^{15}$ I. McCulloch, M. Heeney, C. Bailey, K. Genevicius, I. Macdonald, M. Shkunov, D. Sparrowe, S. Tierney, R. Wagner, W. Zhang, M. L. Chabinyc, R. J. Kline, M. D. McGehee, and M. F. Toney, Nature Mater. 5, 328 (2006).

${ }^{16}$ A. Kikukawa, S. Hosaka, and R. Imura, Appl. Phys. Lett. 66, 3510 (1995).

${ }^{17}$ G. Horowitz, R. Hajlaoui, and P. Delannoy, J. Phys. III 5, 355 (1995).

${ }^{18}$ M. L. Chabinyc, M. F. Toney, R. J. Kline, I. McCulloch, and M. Heeney, J. Am. Chem. Soc. 129, 3226 (2007).

${ }^{19}$ L. M. Andersson, W. Osikowicz, F. L. E. Jakobsson, M. Berggren, L. Lindgren, M. R. Andersson, and O. Inganäs, Org. Electron. 9, 569 (2008).

${ }^{20}$ H. L. Gomes, P. Stallinga, M. Cölle, D. M. de Leeuw, and F. Biscarini, Appl. Phys. Lett. 88, 082101 (2006).

${ }^{21}$ N. Zhao, Ph.D. thesis, University of Cambridge, 2008.

${ }^{22}$ S. G. J. Mathijssen, M. Kemerink, A. Sharma, M. Cölle, P. A. Bobbert, R. A. J. Janssen, and D. M. de Leeuw, Adv. Mater. 20, 975 (2008).

${ }^{23}$ L. Bürgi, T. J. Richards, R. H. Friend, and H. Sirringhaus, J. Appl. Phys. 94, 6129 (2003). 